\title{
BASES SOCIALES DE LA INDIVIDUACIÓN PSÍQUICA EN LA ObRa de G. H. Mead y G. Simondon ${ }^{1}$
}

\section{Social Basis of Psychic Individuation in the Work of $G$. $H$. Mead and G. Simondon}

\author{
David Felipe Villa Bedoya ${ }^{2}$ y John Daniel Londoño Martínez 3 \\ Universidad de Antioquia
}

DOI: $10.17533 /$ udea.rp.v10n1a07

Recibido: 2017-08-12 Aceptado: 2017-10-18

Para citar este artículo en APA: Villa, D., y Londoño, J. (2018). Bases sociales de la individuación psíquica en la obra de G. H. Mead y G. Simondon. Revista de Psicología Universidad de Antioquia, 10 (1), 171-193. DOI: 10.17533/udea.rp.v10n1a07

Resumen: En este artículo se presenta la índole social de la individuación psíquica por medio del análisis de la teoría social de G. H. Mead y el proceso de individuación descrito por G. Simondon. En sus propuestas, ambos autores resaltan la necesidad de comprender la constitución del individuo psíquico como un proceso en constante devenir, mediación entre individuo y sociedad, entre lo interno y lo externo; una singularidad capaz de diferenciarse de otros seres y su entorno, pero en inquebrantable relación con ellos. Se propone una discusión centrada en la pregunta por la naturaleza social de la individuación, principalmente, detallando tres aspectos cruciales para el despliegue de ésta en su fase psíquica: (1) devenir, (2) vínculo individuo-medio y (3) despliegue de potenciales a través de la acción.

Palabras clave: sujeto-objeto, psicología social, devenir, acción, desarrollo.
Abstract: This paper introduces the social nature of psychic individuation based on the analysis of Mead's social theory and the individuation process described by Simondon. Both authors, in their approaches, show the necessity of understanding the individual's psychic constitution as a process of constant becoming, mediation between individual and society, between the inside and the outside; a singularity capable of differentiate itself from other beings and its environment, but in an unbreakable relationship with them. A discussion focused on the social nature of individuation is proposed, mainly, describing three crucial aspects for the deployment of the individuation in its psychic phase: (1) becoming, (2) linkage individual-environment and (3) act and psychic individuation in the social process.

Keywords: subject-object, social psychology, becoming, action, development.

1 Este artículo se desarrolló en el marco de la investigación Bases conceptuales de una psicología de la individuación (Convenio interinstitucional Universidad de Antioquia - Universidad Pedagógica Nacional; códigos: 2017002 [UdeA]; UA-UPN 2017 [UPN]).

2 Estudiante de Psicología en la Universidad de Antioquia. Integrante del grupo de investigación El método analítico y sus aplicaciones en las ciencias sociales y humanas. Correo electrónico: dfelipe.villa@udea. edu.co; https://orcid.org/0000-0002-6423-2973

3 Estudiante de Psicología en la Universidad de Antioquia. Integrante del grupo de investigación El método analitico y sus aplicaciones en las ciencias sociales y humanas. Correo electrónico: johnd.londono@ udea.edu.co; https://orcid.org/0000-0003-4227-9073 


\section{Introducción}

El filósofo francés Gilbert Simondon (1924-1989) expone enlaces entre la filosofía y la psicología a través del concepto de individuación. Con formación en diferentes áreas como la física y la psicología (psicofisiología, psicología comparada, psicología social), su trabajo está enfocado en la descripción del proceso mediante el cual un individuo (molécula, cristal, planta, animal, humano, entre otros) se conforma como devenir constante de información que se amplifica y se organiza como una unidad estructural y funcional (Bardin, 2015; Gil, 2018; Simondon, 2009). En los niveles (1) físico, (2) vital y (3) psíquico y colectivo - las tres fases o dimensiones de despliegue de la individuación-, propone la resolución de problemas como el origen y principal movilizador de la individuación. En la fase psíquica, se introduce lo colectivo en el nexo indisoluble del individuo con su entorno; además, los condicionamientos físicos y biológicos están siempre presentes en la etiología del psiquismo en un entorno cultural (Simondon, 2009, 2013; Ramírez, 2012). Esta fase exige volcar la mirada sobre sí mismo, una búsqueda del entendimiento de la vida psíquica y colectiva para el tránsito a la intersubjetividad y la transindividuación (Simondon, 2009, 2010; Gil, 2016; Builes, Manrique y Henao, 2017).

Al desarrollarse en el marco del proyecto Bases conceptuales de una psicología de la individuación, el presente artículo busca aportar a la construcción de unas bases sociales de la individuación psíquica, cuyo desarrollo esclarezca preguntas concretas del proyecto, a saber: ¿dónde encuentra su raíz el concepto de individuación?, ¿qué caminos introduce para aportar a una psicología contemporánea? El hilo conductor para el establecimiento de unas bases sociales de la individuación psíquica se establece desde dos preguntas guía: ¿qué se entiende por lo psíquico?, ¿cuál es el lugar del otro en dicha constitución? Desde estos cuestionamientos se sustenta la tesis central del actual proyecto de investigación: la individuación psíquica y colectiva acontecen de manera simultánea y ambas conforman la dimensión transindividual. La investigación explora dos dimensiones: epistémica y ascética. La primera se orienta hacia la construcción y formalización de conocimientos; la segunda, a implicaciones 
prácticas -éticas y políticas - para un sujeto en cuanto ser en desarrollo, en devenir dentro de un entorno. De acuerdo con lo anterior, el presente artículo se realiza con el fin de fortalecer la exploración en la dimensión epistémica.

Como punto de partida del proyecto de investigación, y competencia específica del artículo, se identifica la siguiente implicación para la psicología: debe concebirse que individuo y medio son un sistema dentro del cual ocurre la individuación como un proceso; es decir, a medida que se plantean nuevos problemas por parte del mismo individuo o por el entorno (ambiental, cultural, social, político, técnico) que actúa como su complemento, se actualizan los potenciales — preindividuales_-, siempre disponibles para nuevas individuaciones. Así, con el fin de reconocer y resaltar las bases sociales de la individuación psíquica — problema agendado contemporáneamente por Simondon-, se establecen nexos con George Herbert Mead (1863-1931), autor representativo de un enfoque social en psicología.

Este filósofo pragmático, sociólogo y psicólogo social estadounidense fue uno de los fundadores del conductismo social, que posteriormente se consolidó como el interaccionismo simbólico durante el siglo XX. Sus postulados se caracterizaron por señalar al entonces imperante conductismo watsoniano de ser excesivamente simplificador, debido a que había abstraído el segmento del acto del individuo, separándolo del acto completo o social (Morris, 1973). El programa teórico de este autor tuvo como objeto central el estudio de las ciencias de la comunicación y el conductismo; fue considerado como uno de los pragmatistas más distinguidos de la Universidad de Chicago. Sus influencias teóricas conllevaron a comprender que la acción es una operación comunicativa, efectuada en un proceso social puesto en marcha, entre otros, a partir del intercambio de símbolos y significados universales.

Se ha declarado que en la propuesta meadeana del desarrollo de la persona hay una teoría de la individuación (Rendón, 2015; Habermas, 1990). Las características del individuo psíquico se estudian como procesos, más que como estados, y se focaliza el estudio de la mente —o la descripción de los procesos mentales - en el despliegue de los potenciales a través de la acción; esto es, desde la comprensión de Simondon, en el tránsito desde la preindividuación a la individuación y, posteriormente, a la transindividuación. En la teoría del 
"sí mismo" de Mead se encuentra un apoyo para acentuar la índole social de la individuación psíquica como despliegue del ser, en la medida en que el proceso descrito por el autor muestra un importante contraste teórico, una alternativa ante la idea de que el sujeto se constituye como una unidad o identidad aislada; al contrario, incorpora las actitudes de los otros y controla su propia conducta con base en ellas (Rendón, 2015). En sus propuestas, ambos autores resaltan la necesidad de comprender la constitución del individuo psíquico como un proceso en constante devenir, mediación entre individuo y sociedad, entre lo interno y lo externo; una singularidad capaz de diferenciarse de otros seres y su entorno, pero en inquebrantable relación con ellos.

Un proceso de constante tensión, entre otros aspectos, es un punto de partida común entre las teorías de Mead y Simondon: el primer autor describe una tensión entre las fases constitutivas del sí mismo —el 'mí' y el 'yo'(Mead, 2010); el segundo, en términos de información, pormenoriza una tensión interna en el ser individuado, esta es, resonancia de fuerzas energéticas no articuladas, susceptibles de organización que finalmente constituyen la condición de metaestabilidad del ser (Simondon, 2009). Si bien Mead no hace uso explícito del concepto de individuación a lo largo de su obra, Habermas, por ejemplo, ve en sus postulados una teoría de la individuación. En Individuación por vía de socialización: Sobre la teoría de la subjetividad de George Herbert Mead (1990), propone que Mead concibe la individualización social a la luz de la progresiva individuación de la persona; es gracias a la inmersión en relaciones intersubjetivas que se logra la "autodeterminación" y la “autorrealización” (p. 231).

De acuerdo con lo anterior, la individuación requiere, en todo momento, de un entorno social compartido, intersubjetivo, en el cual el sí mismo y su realización se efectúa con los otros. Se propone, entonces, la tesis: la individuación psíquica es inherentemente social, bajo los siguientes argumentos: (1) el desarrollo del individuo psíquico remite a la emergencia como característica esencial de su devenir; (2) el proceso de conformación del individuo acontece en relación a un medio, componen un sistema en el que el encuentro con el otro es fundante del psiquismo y permite a cada uno diferenciarse, poseer una identidad individual y, al mismo tiempo, participar de una dimensión colectiva 
sin caer en la masificación o en la pérdida de singularidad; y, (3) la acción y los procesos psicológicos que la fundamentan —entre otros, pensamiento, lenguaje, reflexión, aprendizaje — son vía para la individuación psíquica y colectiva.

\section{Devenir: permanencia y cambio en el proceso social}

Para Mead (2010), todo individuo se encuentra precedido por condiciones sociohistóricas y culturales componentes del proceso social que, naturalmente, involucran también su propia experiencia. La sociedad se instituye como escenario de interacción entre individuos, intersubjetivo gracias al proceso de comunicación. En el proceso social, comunicativo, las diferentes reacciones que los individuos suscitan entre sí permiten que la acción adquiera significación, por lo que la respuesta conductual del individuo no debe ser entendida como un mero movimiento que carece de finalidad; recurre a los gestos ${ }^{4}$ de los demás y actúa conforme a estos, es decir, son referencia para la acción.

El gesto de un organismo y la respuesta en ajuste de otro organismo a éste, enmarcado dentro de cualquier acto social, revela la relación existente entre el gesto como el comienzo y terminación —o resultante- de dicho acto. Para Mead, el proceso comunicativo es logrado únicamente si el contenido de aquello considerado como referencia es conocido, si se genera un móvil según el cual los gestos de otros se presentan como estímulos con significado

4 El gesto es base del acto comunicativo, se encuentra presente en el comportamiento social del hombre y de animales no humanos. En los últimos, ciertas especies poseen conductas sociales altamente complejas motivadas por el intercambio de reacciones: mediante determinada postura, aullido, aleteo, los animales generan estímulos (gestos) que demandan respuestas de ajuste de los demás miembros de la especie, al ser comprendidos por todos; estímulos externos que propician reacciones generalmente inmediatas. En los seres humanos el gesto es mediado por significados; como estímulo puede ampliarse, variar como término de referencia que conlleva a la acción (Mead, 1974). Una sonrisa puede poseer diferentes significaciones compartidas universalmente: cordialidad, gracia, hipocresía, etc.; además, permite representar el gesto de otro como propio para anticipar las acciones subsecuentes que éste pueda ejecutar. El gesto permite que el ser humano modele programas de acción mediante los cuales puede considerar las reacciones propias como impersonales, con miras a identificar la actuación de cualquier otro individuo frente a estas. En esa medida, el gesto como simbolo significante no es únicamente representación de un estímulo, opera como información que llega a modificarse por la interacción de los individuos y posibilita la "comunicación que está dirigida no sólo a los otros, sino también al individuo mismo” (Mead, 2010, p.170); los significantes son comprendidos por unos y por otros. 
(Miller, 1973). Así, en la acción social hay producción de sentidos en la que intervienen diversos individuos y el gesto se convierte en universal; el individuo se desarrolla bajo los referentes de un sistema psicosocial que, gracias a la simbolización, vale para uno y vale para todos.

Entender de esta manera el proceso social implica que un individuo biológico, dotado en principio de una estructura morfológica y de mecanismos fisiológicos (como un neonato), requiera de la inserción en un marco comunicativo para devenir persona; la experiencia del individuo, como ser social, adquiere un carácter significante. $\mathrm{Al}$ respecto, Morris comenta:

Por medio de un proceso social, pues, el individuo biológico con adecuada materia orgánica obtiene un espíritu y una persona. Por medio de la sociedad el animal impulsivo se convierte en un animal racional, un hombre (...) Así como los primeros niveles del proceso social subsisten después de que se han obtenido los planos superiores, así subsiste el individuo biológico, aun cuando se haya organizado en una persona (1973, p.p. 38-39).

Como resultado, el paso a formas superiores dado en el individuo biológico muestra la importancia de la comunicación significante, la cual radica en la forma en la que el individuo se puede convertir en objeto para sí; el ser humano, a través del proceso social, da cuenta de sí y del medio — es sí y medio simultáneamente- ${ }^{5}$. Mead (2010) postula que la persona se constituye como producto de una confluencia de condiciones singulares y sociales que se manifiesta mediante una interacción continua entre diferentes aspectos que usualmente se entienden por separado, a saber, sujeto y objeto, individuo y sociedad, biología y cultura, permanencia y cambio ${ }^{6}$. En tal confluencia,

5 Mead (1974) propone que la razón no puede convertirse en impersonal a menos de que se dirija hacia una actitud no afectiva, objetiva, hacia sí mismo; de lo contrario, se tiene únicamente conciencia, no auto-conciencia. En su obra resalta la necesidad, para la conducta racional, de que el individuo se convierta en objeto para sí. Para un individuo biológico, entonces, es sumamente importante que la conducta racional intervenga en la situación general donde actúa; es decir, en la perspectiva meadeana, si el individuo no se asume de manera objetiva no puede actuar de manera inteligente, de manera racional.

6 Esta interacción es más que un vínculo entre dos órdenes, magnitudes, dimensiones, que se transforman entre sí; son indisociables, comprenden parte de un mismo sistema. En su encuentro lo fundan, generan un orden nuevo, una emergencia que contiene potenciales de reorganización. El sí mismo comporta interioridad y exterioridad, mismidad y otredad; es propio y ajeno para sí. Se debate en una constante tensión entre individualidad y comunidad, ser particular y común en el tejido social. 
de forma pragmatista, Mead encuentra la autoconciencia como una vía en la que el individuo puede volverse otro de sí mismo, puesto que para planear la acción se convierte en interlocutor de sí: se escucha, se interroga, se responde - como si fuera otro-; esto va más allá de un proceso de abstracción, de un diálogo interno, cuando se modifica la propia acción y el trabajo reflexivo de un individuo se vuelca sobre un público oyente mediante la verbalización (Mead, 1974). El acto se constituye como punto de mediación entre individuo y sociedad, en intersubjetividad; es decir, el paso de la introspección racional a la ejecución es la transición que hace del sí mismo individuo y medio para otros.

Encontramos, entonces, que devenir persona implica la constante interacción en el proceso social. No basta con entender al individuo desde una mera pasividad, esto es, no se reduce a la réplica exacta del proceso que debe interiorizar; menos aún se restringe a la reacción instintiva del organismo en relación con el entorno, a la experiencia o al contagio netamente emocional - experiencia subjetiva- (v.g. las respuestas temperamentales). La comunicación y el pensamiento reflexivo regulan la acción ante el medio; estos procesos dan cuenta de una internalización de la comunicación de gestos en la conducta de un individuo. Adopta las actitudes organizadas de los otros, las cuales serían generadas por la actitud propia, debido a que para planear su acción anticipa las reacciones de los demás y, a tenor de esa reflexión, genera nuevas actitudes en los individuos de la comunidad a la cual pertenece.

Así, los estímulos del medio poseen una doble valencia, son estímulo y respuesta simultáneamente; permiten prever efectos de la acción a realizar. La actividad cognitiva del individuo toma el acto como objeto de análisis y, a través de este proceso, representa su vínculo con el medio, reconociéndose como si para sí y otro para sí. La génesis de los símbolos universales se encuentra en la acción, razón por la cual se otorga un énfasis central a la internalización del gesto y a la reacción ante los estímulos del medio; el mundo simbólico no puede estar al margen de la acción y el pensamiento es, en sí mismo, comunicativo. De este modo, la persona surge en tanto el individuo efectúa un proceso de reflexión fundado en la vivencia intersubjetiva del proceso social; es en sí misma un proceso social. 
Cada vez que la persona enfrenta la problemática práctica, es decir, figura cómo actuar frente al proceso social del que es parte y desde el cual se singulariza, su experiencia se presenta como un movimiento constante entre las disposiciones normativas de la comunidad, las actitudes adoptadas y la actitud particular del individuo como uno diferenciado en sociedad. La constitución de la persona en la interacción intersubjetiva no es lograda únicamente al ligar al individuo al moldeamiento social, a su vez representa el escenario donde se hace expresa su peculiaridad, en el flujo comunicativo reconfigurado en cada intercambio de este con los demás, de este consigo mismo. Emerge allí la tensión entre prescripción social y afianzamiento de la individualidad, una constante oscilación entre dos fases que Mead reconoce como constituyentes de la persona, a saber: yo — de la identidad subjetiva - y mi — de la identidad social- (Rendón, 2015).

El 'yo' en la persona es la respuesta que emite un organismo ante el conjunto — socialmente - organizado de las actitudes de los otros, que este ha interiorizado como 'mí'. El individuo como ser singular se expresa a través del acto de reacción, manifiesta su singularidad en lo colectivo a medida que participa en él. El 'yo' no aparece en el mismo sentido en la experiencia como lo hace el 'mî́: la comunidad de individuos es representada en una organización de las actitudes sociales en las propias actitudes del individuo; ahora, la respuesta que toma lugar — la respuesta del 'yo' - es algo que simplemente ocurre (Mead, 1974).

La operación de la persona sobre un marco social organizado mediante actitudes, que sustentan la expresión espontánea de su singularidad, se desarrolla en una dimensión temporal. Gracias a la representación retrospectiva de sí, el individuo en tanto persona deviene autoconsciente y accede a la posibilidad de que sus experiencias asuman un carácter biográfico, donde el 'yo' y el 'mî' acontecen como memoria de experiencias articuladas en una persona capaz de representar su individualidad.

Hablo conmigo mismo, y recuerdo lo que dije y quizás el contenido emocional que acompañaba lo que dije. El "yo" de este momento está presente en el "mî" del momento siguiente. Y aquí, una vez más, no puedo volverme con suficiente rapidez como para atraparme a mí mismo. Me convierto en un "mî" en la medida 
en que recuerdo lo que dije. Sin embargo, al “yo” puede concedérsele esa relación funcional. Gracias al "yo" decimos que nunca tenemos conciencia plena de lo que somos, que nos sorprendemos con nuestra propia acción. (...) En la memoria, la experiencia del "yo" está constantemente presente. Podemos retroceder directamente unos pocos momentos en nuestra experiencia, y luego, para el resto, dependemos de las imágenes de la memoria. De modo que el "yo", en la memoria, está presente como vocero de la persona en cuanto al segundo, minuto o días pasados (Mead, 2010, p. 202).

Cuando la respuesta toma lugar aparece en el campo de la experiencia, en gran parte, como una imagen de la memoria. Por tanto, existe una separación entre el 'yo' y el 'mí' en el comportamiento y a la vez son partes de un todo (la persona); esto es, "están separados y, sin embargo, les corresponde estar juntos” (Mead, 2010, p. 205). En suma, el 'mî' demanda un 'yo', pero el 'yo' es siempre diferente de lo que la situación misma exige, emerge. Es gracias a estas dos fases, distinguibles, que la persona deviene, se despliega como singular mediante la reacción, la cual alcanza nuevas formas de peculiaridad en la experiencia social al estar en juego con la experiencia individual de los demás, con un 'mí' que no es exactamente igual al de una acción pasada. En ausencia de estas fases no habría novedad en el proceso social.

En la resolución del acto el individuo no pierde cualidad de organismo, comporta una serie de reacciones no deliberadas: una estructura biológica, particular, es condición necesaria para la emergencia de una singularidad. Por consiguiente, en la persona confluyen pasividad y actividad, automatismo y reflexión; ésta es conjunción de experiencias subjetivas y reflexivas. La persona no sólo se capta a sí como individuo en la representación retrospectiva de la reacción del 'yo', también se manifiesta como singular en la visión prospectiva de quién quiere ser (Habermas, 1990). Autoconciencia y pensamiento median las reacciones dispuestas en patrones de actuación ya constituidos, en actitudes previamente asumidas frente a los problemas presentes en el contexto social, dando lugar a una individualidad plasmada en sentido temporal: pasado, presente y futuro confluyen en la autorrepresentación mediante la cual el individuo psíquico se identifica como un ser particular, con una historia propia. Se consolida una "historicidad real" del individuo (Simondon, 2009, p. 
397) en el trayecto o individualización ${ }^{7}$ de pensamientos y reacciones (físicas, corporales), mediante los cuales ha armonizado la tensión presente entre él y el medio, entre el 'yo' y el 'mî', en la resolución de problemáticas, de demandas de vías de acción ajustadas a formas de respuesta previas y a una singularidad constantemente emergente, espontánea (Mead, 2010).

Entre permanencia y cambio se sitúa la individuación, dado que la historia individual de la persona, su constitución como individuo, se encuentra en devenir. Ramírez (2012) resalta la oposición constante entre inmutabilidad y devenir, postulando que el concepto de devenir se contrapone a lo estático, es indicador de cambio y de transformación, designa el paso de unos estados a otros. La concepción del autor es una apuesta en la que se propone una articulación de los procesos cambio y permanencia, los cuales presentan una constante tensión de fuerzas sin resolución aparente: "perdurar no siempre es conservarse, pues se puede llegar al anquilosamiento, la muerte lenta por desadaptación al devenir" (p. 280). El anquilosamiento se correspondería con las nociones de repetición y mismidad si estas últimas excluyeran la posibilidad de una reconfiguración, esto es, si no pudieran cambiar.

La mismidad se refiere precisamente a lo que no cambia, lo que es siempre lo mismo. Sin embargo, en la vida cotidiana, hablamos de que algo se repite cuando es muy similar a otra cosa o evento con el que se lo compara. Es cierto que para que percibamos la repetición debe haber muchos elementos estables, pero no tienen que ser todos: en la práctica, la mismidad no precisa ser absoluta, y de hecho es difícil que pueda serlo. La mismidad no se refiere necesariamente a lo idéntico —en sentido clásico—, sino más bien a la permanencia de elementos, que podríamos llamar esenciales, a partir de los cuales es posible seguir reconociendo algo a pesar de que muchos de sus aspectos se hayan modificado por efecto de distintas intervenciones (Zuluaga, 2014, p. 23).

El proceso social, por su parte, no puede encontrarse al margen de este devenir, constituye la confluencia en la que se encuentra el individuo con el

7 Para Simondon (2009), el proceso de individualización es aquel cuando un ser, en principio individuado, encuentra nuevas formas de individuación. El individuo psíquico y colectivo adquiere una singularidad, su identidad gradualmente se configura y consolida tras el constante despliegue de potenciales. Cada momento de individuación, de desfase del ser, produce un cúmulo de huellas que conforman una historia singular, "lo que cada uno es en cuanto individuo con un deseo singular que, se espera, esté en conformidad con el entorno del cual es parte" (Gil, 2016, pp. 65-66). 
entorno y con la sociedad; permite, entre otras razones, evidenciar una influencia que no lleva de manera inminente a una mera recreación de los acontecimientos sociales (Rendón, 2015). En otras palabras, el hecho de que los procesos sociales sean potenciadores de las pautas de conducta no implica, en todo momento, la plena repetición de dichos patrones. La persona, asimismo, contiene en sí un proceso de constitución integradora de identificación y diferenciación consigo y con el otro, emerge en el devenir constante del proceso social; tensión entre permanencia y cambio, donde "la emergencia involucra una reorganización, pero la reorganización introduce algo que no existía antes" (Mead, 2010, p. 223).

\section{La individuación psíquica: vínculo individuo-medio}

El proceso social es el campo de despliegue del individuo en tanto las vivencias se encuentran mediadas por la organización social que antecede a la conformación de una individualidad. Ningún individuo que pretenda consolidarse en una posición particular dentro de la sociedad puede sustraer el carácter social, simiente e inexorable, de dicha conformación; en la interacción con el medio el individuo adquiere una historia que constituye su singularidad como articulación de experiencias.

Por otra parte, si se plantea que los criterios sociales no restringen la experiencia del individuo a una réplica de aquello establecido como normativa, en un grupo social determinado, cabe preguntar: ¿cómo acontece la relación individuo-entorno?; asimismo, ¿cómo puede el individuo sobreponerse a los marcos de acción estipulados como socialmente objetivos, legítimos? En el carácter de la relación individuo-entorno no puede haber una primacía inherente de uno de estos componentes, una unidireccionalidad; individual y social son un sistema, comportan un vínculo indisociable entre sí (Simondon, 2009).

Los planteamientos de Simondon permiten esclarecer esta indagación: la pregunta ontológica sobre el ser debe virar de la comprensión de este como sustancia, a la concepción del individuo en tanto expresión de un proceso de reorganización constante —ontogénesis_- (Vargas, 2014; Simondon, 2009). 
Para estudiar la individuación, Simondon (2009) recurre al problema de cómo los seres devienen singularidades en un medio compartido con otros seres. Ahora bien, la pregunta por el ser se encuentra en el proceso en el que aquel está siendo, es decir, en su devenir. Esta concepción, en la cual ser y devenir no son nociones contrapuestas, conduce al planteamiento del ser como individuo; el cual es un modo o "momento de ser" (2009, p. 477).

Este autor concibe un estado anterior a cualquier heterogeneidad: un todo indiferenciado como condición primigenia de la naturaleza. Más que entrópica ${ }^{8}$, esta resguarda potenciales y deviene en escenario de emergencia; contiene la capacidad de diferenciación y complejización que permite la multiplicidad y mutación de esa indistinta energía primaria en seres, singulares constantemente desplegados como una organización particular al expresarse como individuos. Así, todo individuo precisa como antecedente una realidad preindividual, aquel escenario indeterminado de fuerzas energéticas capaces de articularse en organizaciones estructurales y funcionales:

El individuo sería captado entonces como una realidad relativa, una cierta fase del ser que supone antes que ella una realidad preindividual y que, aún después de la individuación, no existe completamente sola, pues la individuación no consume de golpe los potenciales de la realidad preindividual, y por otra parte, lo que la individuación hace aparecer no es solamente el individuo sino la pareja individuo-medio (2009, p.26).

El proceso de individuación se genera cuando los potenciales - flujo de energía- asumen carácter de información, conforman una organización singular que entra en resonancia con un medio y deviene en una estructura funcional concreta por ser la resolución a un problema (Simondon, 2009); una presión conducente al despliegue o realización de aquellos potenciales. Cuan-

8 Todo sistema tiene una tendencia natural a ser entrópico de acuerdo con la segunda ley de la termodinámica: en él permanecen niveles de desorden y aleatoriedad que alcanzan el grado máximo cuando el sistema se encuentra alejado de su ambiente; reduce o pierde el intercambio de información con él. La información, comprendida desde la teoría matemática de la comunicación (Shannon, 1948), es entropía negativa (neguentropía), puesto que restablece la organización del sistema constantemente. Lo viviente opera por códigos de información, gracias a ellos se desarrolla como un sistema, un orden de energía y materia, en un proceso continuo de diferenciación con su entorno (Huneeus, 1978). Para su diferenciación como individuo, el ser requiere de información que comunica energía y actualiza potenciales (Simondon, 2009): la neguentropía es principio de individuación. 
do el estado preindividual, indiferenciado, logra ordenarse en determinada estructura, esta persiste como realidad organizada, mas no por ello pierde la posibilidad de complejizarse: conserva potenciales preindividuales. Así, el ser emerge como individuo y se amplifica en la obtención de estabilidad transitoria, dado que está sujeto a conservarse y cambiar, de manera simultánea. Para Simondon, la individuación es operación transductiva ${ }^{9}$, de modulación y, a su vez, de organización. La primera es ampliación mediante el reordenamiento, desfase del individuo que rebasa un estado previo y se despliega como más de sí; la segunda es garante de la permanencia, conservación de la estabilidad que el individuo alcanza tras cada momento de cambio (Gil, 2016).

El proceso de amplificación, entonces, es operación organizativa - $\mathrm{u}$ organizadora - en la articulación entre estatismo y dinamismo: la existencia del individuo es devenir entre lo constituido y aquello que potencialmente emerge como nueva organización. El ser no es definitivo ni eterno, es un sistema configurado en el medio a través de subsecuentes momentos de ajuste. El individuo es expresión de resolución de problemas, a partir de ello deviene en nuevas organizaciones estructurales y funcionales que resguardan una historia de adaptaciones; la resolución implica un proceso de constante desfase, un llegar a ser más de lo que es, en el recuento de individuaciones consolidantes de la singularidad, de la individualización.

En los sistemas que alcanzan formas novedosas de operación, la metaestabilidad es propiedad necesaria. Los estados de equilibrio y armonía siempre son parciales, conducen a la posible obtención de estados más estables; de modo que, la emergencia del ser individuado es constante. Las diferentes formaciones del ser, como expresión resolutoria de un problema, nunca se agotan dado que están en permanente relación con un medio; coexisten armonía y capacidad de cambio. La metaestabilidad es motor de individuación en tanto constituye el alcance de un estado del ser como individuo individuándose: se presentan así estados previos de organización, sin ello el ser nunca podría

9 La transducción es una operación estructurante a nivel físico, biológico, mental y social, en la medida en que permite la constitución de nuevos componentes en cada uno de estos dominios. Una estructura dada sirve de escenario de conformación de nuevos órdenes estructurales; el movimiento de ampliación se propaga y cada organización alcanzada es germen de estructuración para nuevos órdenes: "una estructuración del dominio operada aquí y allá" (Simondon, 2009, p. 38). 
llegar a configurarse como unidad diferenciada; pero, a su vez, no se da un agotamiento total de los potenciales, de la energía remanente que permite el despliegue del ser individuado y el alcance de otro estado metaestable. Por tanto, la individuación es ontogénesis del ser bajo condición de metaestabilidad.

No sólo es el medio quien comporta un carácter preindividual, el individuo en sí mismo contiene una carga preindividual que es necesaria para la puesta en marcha de la operación de amplificación. Se presenta una resonancia interna en el ser individuado, esta es, campo de fuerzas energéticas no articuladas, susceptibles de organización que, finalmente, constituyen la tensión de metaestabilidad del ser (Simondon, 2009). De tal modo, la carga preindividual hace parte de un sistema: individuo-medio, "el medio, por otra parte, puede no ser simple, homogéneo, uniforme, sino estar originalmente atravesado por una tensión entre dos órdenes extremos de magnitud que el individuo mediatiza cuando llega a ser" (Simondon, 2009, p. 26). El ser, en tanto individuo, emerge a partir de los potenciales energéticos de un medio, razón por la cual permanecerá asociado a este.

La individuación, para Simondon (2009), se desarrolla en tres fases, a saber: (1) física, (2) biológica o vital y (3) psíquica y colectiva — transindividual - En cada una de éstas emergen problemas diversos en el sistema individuo-medio; superarlos dirige a un desfase de sí, del medio, a un nuevo momento de individuación. Existe, pues, un tránsito entre individuo físico, biológico y psíquico; en cada fase posee una estructura más compleja para solventar las problemáticas del sistema: la individuación psíquica subsume las fases vital y física en una nueva forma de organización — estructura y función-, somatopsíquica.

El psiquismo aparece como una nueva capa de individuación del ser, que tiene por correlato, en el ser, una incompatibilidad y una sobresaturación ralentizante de los dinamismos vitales, y fuera del ser en tanto individuo limitado, un recurso a una nueva carga de realidad preindividual capaz de aportar al ser una nueva realidad; lo viviente se individúa más precozmente, y no puede individuarse siendo su propia materia, como la larva que se metamorfosea al nutrirse de sí misma; el psiquismo expresa lo vital y, correlativamente, una cierta carga de realidad preindividual (Simondon, 2009, p. 243). 
En consecuencia, de acuerdo con lo desarrollado en el apartado anterior, el tránsito de niveles inferiores a formas superiores de funcionamiento no es causa de su extinción; los primeros niveles se preservan como condiciones base para la conformación de nuevas organizaciones, siguen operando en ellas. De esta manera, niveles primarios del proceso social (gesto animal) permanecen aún después de que niveles superiores son obtenidos (símbolo significante). Asimismo, el individuo biológico permanece cuando se ha organizado en una persona, tal como el individuo psíquico es conjunción de condiciones físico-biológicas. Ahora, se entiende lo psíquico como un orden nuevo debido a que emerge de la interacción entre lo biológico-hereditario y lo social-cultural como fundante del aspecto simbólico y discursivo (Lopera, Manrique, Zuluaga y Ortiz, 2010). Es reconocida la existencia de una misma realidad: el psiquismo es resultante de dos dimensiones, biológica y cultural, que interactúan entre sí y devienen en un sistema más complejo.

El átomo, el cristal, la larva, el ser humano, son individuos; si bien difieren en estructura y función, se conforman a través de un mismo proceso: devenir entre permanencia y cambio. La carga preindividual y la metaestabilidad son condiciones comunes a cada uno de ellos; potenciales de amplificación en el sistema individuo-medio. La fase psíquica y colectiva transcurre en confluencia de individuos autoconscientes, reflexivos, con posibilidades de decisión para la acción; en último término, como teatro de individuación que maximiza potenciales.

El ser humano, como sujeto ${ }^{10}$ psíquico, es capaz de dar cuenta de sí, de cómo está siendo; puede dirigir su proceso de individuación, partiendo de un hecho, se percibe a sí mismo y en relación con otros. Gracias a capacidades cognitivas complejas logra autorrepresentarse; procesos como la conciencia, el lenguaje, el pensamiento, el aprendizaje, dan pie a una capacidad creativa: "[es] el más rico en posibilidades de individuación, pues nunca está acabado, sus opciones se despliegan a medida que crece" (Gil, 2016, p. 57).

10 El sujeto (psíquico) es el viviente capaz de representar su acción como operación que se dirige al mundo y se dirige a sí mismo como representación del mundo. Por medio del acto el sujeto interviene en el entorno; antes de hacerlo lo comprende, lo toma para sí, sabe de él para poder desplegar su acción. Los problemas vitales no se confinan en el sujeto, su individuación supone una realidad preindividual que lo incorpora en relación con el medio a través de la resolución de potenciales propios y del mundo (Simondon, 2009). 
Para el ser humano la metaestabilidad sólo logra alcanzar su mayor potencial en lo colectivo; sólo en vínculo con otros individuos psíquicos puede acceder al despliegue de toda la energía remanente del sistema, que le permite encontrar nuevas formas de ser en el medio. Su individuación toma lugar en interdependencia; la carga preindividual de los demás seres que constituyen su entorno se hace necesaria para alcanzar estados de ampliación como sistema: no es posible concebir al ser como distinto al medio con el cual se encuentra asociado (Simondon, 2009); luego, si dicho entorno es esencialmente social, el humano no puede ser ni llega a ser más de sí por fuera de la organización y proceso social, sin el establecimiento directo de vínculos con los demás (Mead, 2010). Lo colectivo es condición necesaria para la resolución de problemáticas y actualización de potenciales, el constante alcance de una mejor organización de ser individuado, lo que es, "(...) individuaciones que resuelven el mundo y resuelven el ser individuado” (Simondon, 2009, p. 322).

En la interacción entre sujetos psíquicos se construye lo transindividual, puesto que los individuos, aunque diferenciados, se relacionan de forma tal que la individuación de un sujeto contempla la de otros. Se establece una dimensión más allá de los individuos: el nosotros, donde la amplificación de la individuación se da entre estos, necesita de ellos para su operación (Gil, 2016). La colectividad es fundante de lo transindividual, cuando en ella el individuo deviene individuo de grupo, no solamente es sujeto en un contexto social o perteneciente a relaciones interindividuales; bajo estas condiciones los individuos establecen entre sí vínculos, pero no necesariamente con el fin de transformarse entre ellos. El sujeto psíquico deviene transindividual cuando su individuación toma lugar en el momento que esta es dada en los demás individuos, miembros de un grupo de pertenencia. Los demás no son accesorios, no se encuentran restringidos a facilitar nuevos momentos de individuación; más que ello: el desfase del ser como individuo psíquico requiere, ineludiblemente, de una relación constitutiva con el otro. 


\section{La acción: vía de la individuación psíquica en el proceso social}

¿Cómo se desenvuelve el sujeto en la esfera social? El desarrollo del individuo psíquico supone la pregunta por su despliegue tras cada momento de individuación, por su proceso de ser, de formar-se. Las tensiones entre el individuo y su entorno confluyen a través de la acción, se articulan o armonizan de manera parcial mediante la ejecución del individuo. El esquema estímulo-respuesta es necesario, mas no suficiente para comprender la acción; parte de ésta tiene lugar al interior del individuo no sólo como procesamiento del estímulo, permite la anticipación del acto aun cuando no va a ser llevado a cabo de forma consecutiva: sobre la representación de la realidad se planifica la ejecución, se anticipa a su materialización, pero no todo programa de acción pensado se expresa de inmediato en la medida en que puede retornar, posteriormente, al medio (Mead, 1938, 1974). A través del pensamiento se organiza la realidad, se discierne entre las relaciones que la constituyen $y$, mediante aquel discernimiento, el individuo psíquico tiene un vasto número de posibilidades para ser y estar en el entorno (Vygotsky, 1995; Mead, 2010).

El pensamiento, como proceso, es acción comunicativa gracias al lenguaje - constitución de significantes - en tanto opera sólo a partir de la comunidad, de la intersubjetividad donde se construyen contenidos con sentido (Mead, 2010); los gestos universales permiten representar el mundo, mediar la interacción (Miller, 1973). En la dupla individuo-medio, la acción es bisagra entre lo particular y lo universal, sustento de dicho sistema; de hecho, la conformación misma del contenido del pensamiento de un individuo está siempre vinculada con el cúmulo de experiencias de su ontogenia. Así, a partir de la confluencia de determinada carga biológica, y de aquello que proviene de la realidad externa, se da la forma y el contenido del pensamiento y lo internalizado es llevado afuera por medio de la acción: modifica la realidad misma de la cual surgió en principio (Rivière, 1984).

Tomar la acción como punto de partida, como mediación, es esencial para entender el sistema individuo-medio; la reflexión lo es, en igual medida, para 
el desarrollo de la persona y el despliegue de la individuación psíquica. Sin embargo, lo anterior no conlleva a que estos procesos incurran en una negación de aquellos no reflexivos. La acción no es exclusivamente respuesta o reflejo, implica transformación del medio dentro del cual está incluido el propio individuo. El concepto de actividad está estrechamente relacionado con el de mediación, la cual permite no solamente la conformación de las singularidades de un sistema, también la regulación y transformación del medio externo, es decir, entender la reflexión para la acción comprende una doble vía: de afuera hacia adentro y de adentro hacia afuera.

Actuar reflexivamente en un entorno, como vía de acople, potencia la constitución, recepción y reformulación de conocimientos que posibilitan captar las condiciones del medio y disponer de estrategias adaptativas en él. Es mediante ese doble movimiento que el individuo se distancia de sí mismo y del entorno, a través de la representación, la abstracción y la mediatez cognitiva ${ }^{11}$ (modelamiento de distintos programas de acción), siempre para regresar a dicho entorno mediante la materialización del pensamiento en la ejecución: a medida que el individuo es capaz de tomar la realidad como objeto de su pensamiento la descifra con mayor detalle, amplifica su capacidad de transformación (Vygotsky, 1995).

El sistema individuo-medio, y la individuación que acontece en relación, remite a una concepción en la cual el individuo se presenta como sujeto y objeto simultáneamente, en la medida que este es medio singularizado y el medio es ser individuado:

Debe quedar claro a estas alturas que ni el sujeto corresponde al organismo, ni tampoco puede el objeto concebirse como el supuesto 'contenido' del conocimiento de un sujeto. La filosofía de la individuación se ocupa más bien del sistema de potenciales en los que la operación de conocimiento surge como una relación sujeto-objeto (Bardin, 2015, p.3).

11 El lenguaje, primordial para el funcionamiento de la mente y la reflexión, posibilita representar la realidad a través de mediadores como los símbolos y las palabras, los cuales son formas de expresión de mediatez cognitiva, de la capacidad de postergar y analizar las exigencias inmediatas del ambiente. Tal distanciamiento da lugar, entre otras razones, al alcance del aumento de la eficacia de la adaptación: el control. La mediatez permite que la conciencia no se limite a los procesos sensoperceptivos dados en la interacción inmediata con el ambiente (Chávez, 2011). 
Un individuo pasa a ser él mismo a través de los otros, esto es, su personalidad viene a ser para sí lo que es en sí — consolidación de una historia individual - a través de las significaciones que conforma con los demás. Existe una constante interrelación del sujeto con el objeto en cada momento de resolución de problemáticas, en el entre del individuo y del medio se constituye un escenario de intercambio de acción y significados: sujeto y objeto se configuran en ese encuentro, pues la operación del conocimiento y la ejecución del acto transforman al medio y transforman al individuo. Con ello adviene una implicación epistemológica, la díada sujeto-objeto se funde en un sistema donde el individuo no se limita a ser un observador que capta la realidad externa en una representación interna; tampoco debe entenderse al individuo como único rector y creador de su realidad, esto conlleva al establecimiento de una relación causal que va de manera exclusiva desde factores internos atribuidos al individuo (v.g. la reflexión), para después volcarse sobre la realidad y modificarla de manera unidireccional — de adentro hacia afuera—. Al encuentro con los objetos, el sujeto los reconfigura como aquel entorno del que se sabe partícipe y en el cual despliega su ser; es decir, es individuado por el medio.

No toda acción del sujeto psíquico se desprende de procesos reflexivos. Las reacciones organísmicas, temperamentales, emocionales, continúan presentes; ante una amenaza el individuo puede enfrentarla, paralizarse o huir, sin antes considerar un curso adecuado de acción. Olvidar las disposiciones biológicas del individuo psíquico para adaptarse en el medio implicaría incurrir en un error. Del mismo modo lo sería desconocer la automatización de aprendizajes y ejecución de ciertos patrones de acción. Por ejemplo, quien conduce hace diez ańos no invierte el mismo esfuerzo cognitivo que aquel que se encuentra en sus primeras clases de conducción; un proceso reflexivo, autoconsciente, puede convertirse en hábito, llegar a operar de manera implícita. Niveles primarios y superiores no se excluyen ni sobreponen; el desarrollo de los procesos mentales, mediante el ingreso a las instituciones, el acceso al arte, al uso de objetos técnicos, etc. (i.e. a la cultura), reorganizan las disposiciones biológicas y conforman un nuevo sistema: el psiquismo. El pensamiento reflexivo repercute en el proceso de formación del sujeto psíquico; representar la acción como 
operación que se dirige a sí mismo y al mundo permite que el acto del sujeto, aquel que interviene en el entorno y organiza el sistema individuo-medio, sea controlado. Anticipar un acto antes de ejecutarlo implica que el individuo pueda dirigir parte de su proceso de individuación. El margen de acción se amplía.

En definitiva, la acción es primordial para pensar la transformación del individuo y del medio, es decir, la regulación y modificación del entorno y de la conducta se da en constante mediación —sujeto-objeto-, en la comunicación en el proceso social. La acción es esencialmente intersubjetiva, "es este intercambio en red entre los individuos de un colectivo, intercambio que crea la resonancia interna [el potencial de ampliación] del conjunto formado" (Simondon, 2009, p. 327). Mediante el acto se resuelven parcialmente tensiones entre el individuo y el medio; en encuentro con los otros se superan problemáticas y, en relación con ellos, se accede a nuevos momentos de individuación.

\section{Conclusión}

Con el solo hecho de que se practique la psicología social estamos saliéndonos de la ontología objetivista y no podemos quedarnos allí sólo ejerciendo sobre el "objeto", poniéndonos un limite que compromete la investigación ${ }^{12}$

Maurice Merleau-Ponty

La dimensión transindividual es psicosocial. La significación emerge en el intercambio comunicativo, operación colectiva de formación de sentidos, de emergencia de símbolos significantes en una unidad social donde la relación intersubjetiva no es simple contacto, es transformación. Cuando el individuo representa un símbolo recíproco para otros sujetos (Heredia, 2015, p. 459) el flujo de información, garante de individuación, se amplía en la confluencia de

12 Traducción nuestra. 
los potenciales preindividuales compartidos con los demás. El sujeto ya no se corresponde con la idea de un ser sustancial: es relación, devenir de la realidad psicosocial.

Lo colectivo es más que relación entre individuos, es base para la conformación del sujeto como singularidad, la cual es expresión de autoconciencia, de la capacidad que funda el distanciamiento de la esfera de la experiencia directa del mundo, para pensar sobre sí; pero, también para ir a la experiencia misma, analizarla, detenerse sobre ella. Lo inmediato se convierte en mediato; el hombre encuentra diversos cursos de acción, de ser y hacer en el medio, en tanto no está plenamente limitado a una respuesta automática a las presiones físicas y socioculturales de su entorno: el individuo psíquico resuelve problemas y los crea, responde y a su vez transforma el medio que, en último término, es un mundo de vida compartido.

La mediación entre lo psíquico y lo colectivo está irresuelta si el campo de estudio de la psicología y la sociología se remite de manera exclusiva al individuo y a la sociedad, respectivamente; si no se logra un real entendimiento de dicha mediación. Esto se debe, por una parte, a que se aíslan características que, con frecuencia, impiden el abordaje de una realidad compleja y la consolidación de una teoría general; por otra, los intentos de buscar acuerdos y puntos medios suponen la amenaza de caer en un eclecticismo que superpone conceptos, o bien, que suma lo 'mejor' de cada disciplina generando un abordaje que, aunque dialéctico, llega a resultar insuficiente (Heredia, 2015).

La pregunta por la naturaleza social de la individuación psíquica, desde los autores G. H. Mead y G. Simondon, remite al proceso de desarrollo de un sujeto en devenir, inmerso en interacciones sociales que configuran el entorno en el proceso de individuación; se genera un ir y venir constante que, por medio de la acción, no sólo individúa al sujeto, sino que individúa al medio. La individuación psíquica es inherentemente social puesto que es en intersubjetividad donde aquello que podría considerarse como lo más individual, privado (v.g. la personalidad, la identidad) se construye con otros. Referirse al sistema individuo-medio encamina nuevas preguntas no sólo para el horizonte del actual programa de investigación en individuación, indaga por las bases de una psicología que pueda ser entendida, en términos generales, como social. 


\section{REFERENCIAS}

Bardin, A. (2015). Epistemology and political philosophy in Gilbert Simondon: Individuation, Technics, Social Systems. Londres: Springer.

Builes, I., Manrique, H. y Henao, C. (2017). El proyecto simondoniano: la individuación del ser en devenir. Revista Co-herencia, 14(26), 177-205.

Chávez, L. (2011) La inteligencia social y sus implicaciones en la evolución de la mente. Revista de Psicología Universidad de Antioquia, 3(1), 73-86.

Gil, L. M. (2016). Psicología, trabajo e individuación. Bogotá: San Pablo.

Gil, L. M. (2018). Psicogénesis y sociogénesis: Psicología social e individuación en la obra de G. Simondon. En Autora. Psicología de la individuación. Bogotá: Aula de Humanidades.

Habermas, J. (1990). Pensamiento postmetafísico. México: Alfaguara.

Heredia, J. (2015). Lo psicosocial y lo transindividual en la obra de Gilbert Simondon. Revista Mexicana de Sociología, 77(3), 437-465.

Huneeus, F. (1978). Una fundamentación científico natural del concepto de Gestalt. En Stevens, O. (Comp.), Esto es Gestalt (pp. 311-327). Santiago de Chile: Editorial Cuatro Vientos.

Lopera, J. D., Manrique, H., Zuluaga, M. y Ortiz, J. (2010). El objeto de la psicología: el alma como cultura encarnada. Medellín: Universidad de Antioquia.

Mead, G. H. (1938). The Philosophy of the Act. Chicago: University of Chicago Press.

Mead, G. H. (1974). Mind, Self, and Society. Chicago: University of Chicago Press. 
Mead, G. H. (2010). Espiritu, persona y sociedad. Desde el punto de vista del conductismo social. Madrid: Paidós.

Miller, D. (1973). George Herbert Mead: self, language and the world. Texas: Library of Congress Cataloging in publication data.

Morris, C. (1973). George Herbert Mead como psicólogo y filósofo social. Introducción a Georg. H. M. Espíritu, persona y sociedad. Barcelona: Paidós.

Ramírez, C. (2012). La vida como un juego existencial. Ensayitos. Medellín: EAFIT.

Rendón, C. E. (2015). De la igualdad de los derechos al derecho a la diferenciación. La idea de la democracia en G. H. Mead. Revista Latinoamericana de Filosofía Política, 4(2), 1-29.

Rivière, A. (1984). La psicología de Vygotski: sobre la larga proyección de una corta biografia. Madrid: Visor Infancia y Aprendizaje.

Simondon, G. (2009). La individuación a la luz de las nociones de forma y de información. Buenos Aires: Cactus y La Cebra.

Simondon, G. (2010). Comunicación e información. Buenos Aires: Cactus.

Simondon, G. (2013). Imaginación e invención (1965-1966). Buenos Aires: Cactus.

Vargas Guillén, G. (2014). Individuación y anarquía: metafísica y fenomenología de la individuación. Bogotá: Editorial Aula de Humanidades.

Vygotsky, L. S. (1995). Pensamiento y lenguaje. Obras escogidas (Tomo II). Madrid: Visor.

Zuluaga, M. (2014). Identidad y devenir. Bogotá: San Pablo. 
